\title{
Resource Allocation in Nonlinear Flexible-Grid Fiber-Optic Networks
}

\author{
Li Yan, Erik Agrell, and Henk Wymeersch \\ Department of Signals and Systems, Chalmers University of Technology, SE-41296 Gothenburg, Sweden \\ lyaa@chalmers.se
}

\begin{abstract}
We describe a novel method for joint resource allocation in flexible-grid networks based on a nonlinear impairment model. Compared with the fixed-grid WDM benchmark, our algorithm achieves $38 \%$ bandwidth saving and significant transmission distance extension.
\end{abstract}

OCIS codes: (060.4510) Optical communications, (060.4256) Networks, network optimization.

\section{Introduction}

Motivated by the exponentially increasing Internet traffic, flexible-grid optical networks are actively studied to make more efficient use of the optical bandwidth [1]. To account for inter- and intra-channel interference in such networks, a realistic, yet tractable physical layer impairments (PLI) model is needed. Previous approaches to estimate PLI relied on transmission reach [1], which is not capable to fully characterize the nonlinear interference (NLI). More sophisticated analytic PLI models were proposed recently [2,3]. Based on these models, [3,4] demonstrated overall network throughput improvement by optimizing channel power and modulation format for fixed-grid WDM networks. Flexible-grid superchannels were considered in [5,6], where channel power or channel spacing were separately optimized. However, such separate optimization cannot fully harness the advantages of the flexible-grid network. In this paper, we propose a novel joint optimization approach of channel power spectral densities (PSDs), carrier frequencies, and modulation formats, in order to minimize the bandwidth in nonlinear flexible optical networks. The numerical results show great improvement in terms of bandwidth utilization and transmission distance extension over the state of the art $[3,4]$.

\section{Physical Layer Model}

We use the Gaussian noise (GN) model [2] to estimate the PLI and calculate signal-to-noise ratio (SNR). The GN model is an approximate quantitative model for NLI that allows calculation of the SNR for all channels in a link [2]. In this model, we assume (i) a coherent polarization-multiplexed system without inline compensation for chromatic dispersion; (ii) for each channel, the transmitted signal PSD is equal in both polarizations; (iii) each channel spectrum is rectangular and does not overlap with neighboring channels; (iv) the NLIs generated in different fiber spans sum incoherently over the whole link; (v) the loss of each fiber span is compensated by an EDFA at the end of the span; (vi) the length of each span is equal; and (vii) the self-phase modulation is fully mitigated by suitable receiver design [3]. Under the assumptions above, the NLI PSD for channel $i$ on one polarization can be expressed as [2, Eq. (16)]

$$
G_{i}^{\mathrm{NLI}}=\sum_{l \in P_{i}}\left(\mu N_{l} G_{i} \sum_{j \in C_{l}} G_{j}^{2} \ln \left|\frac{\left|f_{i}-f_{j}\right|+\Delta f_{j} / 2}{\left|f_{i}-f_{j}\right|-\Delta f_{j} / 2}\right|\right)
$$

where $P_{i}$ is the set of links on the light path of channel $i, C_{l}$ is the set of channels on link $l, N_{l}$ is the number of spans in link $l, \mu=3 \gamma^{2} /\left(2 \pi \alpha\left|\beta_{2}\right|\right), \gamma$ is the nonlinear parameter, $\alpha$ is the fiber power attenuation, $\beta_{2}$ is the group velocity dispersion (GVD) parameter, and $\Delta f_{k}, f_{k}$, and $G_{k}$ are the $k^{\text {th }}$ channel's bandwidth, center frequency, and signal PSD on one polarization, respectively.

In addition to the NLI, each EDFA adds white Gaussian noise with PSD $G_{i}^{\mathrm{ASE}}=\left(e^{\alpha L}-1\right) h v n_{\mathrm{sp}} \sum_{l \in P_{i}} N_{l}$, where $L$ is the fiber length per span, $n_{\mathrm{sp}}$ is the spontaneous emission factor, $v$ is the light frequency, and $h$ is Planck's constant. Combining the linear and nonlinear noises, the SNR for channel $i$ can be approximated as $\mathrm{SNR}_{i}=G_{i} /\left(G_{i}^{\mathrm{ASE}}+G_{i}^{\mathrm{NLI}}\right)$. In this paper, we use $\alpha=0.22 \mathrm{~dB} / \mathrm{km}, \gamma=1.3 \mathrm{~W}^{-1} \cdot \mathrm{km}^{-1}, \beta_{2}=-21.3 \mathrm{ps}^{2} / \mathrm{km}, L=100 \mathrm{~km}$, and $n_{\mathrm{sp}}=1.58$, which are all the same as those in our benchmark [4].

\section{Problem Statement and Optimization Formulation}

In this paper, following the same decomposition strategy in [3,4], channel routing and channel ordering per link are assumed predetermined. Given the routing of every channel and the ordering of channels on each link, we can then optimize their modulation formats, carrier frequencies, and PSDs to minimize the bandwidth used by the network. We denote the set of channels by $\mathbf{T}$, the set of links in the network by $\mathbf{E}$, the $i^{\text {th }}$ channel's data rate by $R_{i}$ (in Gbps), and its PSD as $G_{i}$ (in $\mu \mathrm{W} / \mathrm{GHz}$ ). The spectral efficiencies of available constellations are $c_{i} \in \mathscr{C}$, where $\mathscr{C}$ is a finite set $\left(\mathscr{C}=\{2,4,6,8,10,12\} \mathrm{bit} / \mathrm{s} / \mathrm{Hz}\right.$ in this paper). Thus the bandwidth of channel $i$ using constellation $c_{i}$ is $\Delta f_{i}=R_{i} / c_{i}$ 
(in $\mathrm{GHz}$ ), assuming Nyquist pulse shaping. The center frequency of each channel is assumed flexible (no frequency grid). To obtain a satisfactory quality of transmission, $\operatorname{SNR}_{i} \geq \operatorname{SNR}_{\text {th }}\left(c_{i}\right)$ is required for all $i \in \mathbf{T}$, where $\operatorname{SNR}_{\text {th }}\left(c_{i}\right)$ is the SNR threshold (in linear scale) required to obtain a certain BER (chosen to be $4 \times 10^{-3}$ in this paper, as in [4]). The resource allocation can be formulated as

$$
\begin{array}{clc}
\underset{\mathbf{c}, \mathbf{f}, \mathbf{G}, u, \mathbf{t}}{\operatorname{minimize}} & \theta_{1} u+\theta_{2} \sum_{i} \frac{R_{i}}{c_{i}} G_{i}+\theta_{3} t_{m} & \\
\text { subject to } & t_{i} \cdot \mathrm{SNR}_{i}(\mathbf{c}, \mathbf{f}, \mathbf{G}) \geq \operatorname{SNR}_{\mathrm{th}}\left(c_{i}\right), & i \in \mathbf{T} \\
& K_{j}(\mathbf{c}, \mathbf{f}) \leq 0, & j \in \mathbf{E} \\
& u \geq \frac{R_{i}}{2 c_{i}}+f_{i}, & i \in \mathbf{T} \\
& t_{m} \geq t_{i}, & i \in \mathbf{T},
\end{array}
$$

where $K_{j}(\mathbf{c}, \mathbf{f})$ are constraints that guarantee the neighboring channels do not overlap in spectrum (note that the channel spacing can be any preset value according to the wavelength selective switch (WSS), and the algorithm will give the corresponding solution.); $u$ is the upper bound of the occupied frequency; $t_{i}$ is the inverse of the SNR margin in linear scale for channel $i$, and $t_{m}$ represents the inverse of the minimum SNR margin. The objective function in (3) comprises three separate objectives, which can be tuned using the weighting factors $\theta_{i} \geq 0$. In particular, $\theta_{1}>0, \theta_{2}=\theta_{3}=0$ aims to minimize total bandwidth utilization, $\theta_{2}>0, \theta_{1}=\theta_{3}=0$ aims to minimize the total transmit power and thus decrease the nonlinearity of the fiber, and $\theta_{3}>0, \theta_{1}=\theta_{2}=0$ aims to maximize the minimum SNR margin. Note that this latter case was solved in [4] for a fixed WDM grid with fixed modulation formats.

\section{Numerical Results}

To demonstrate the performance of the proposed optimization formulation, we compare with the fixed-grid network planning approach from [4], considering the same three-node network (shown in Fig. 1). The benchmark uses a fixedgrid WDM-based nonlinear PLI model, which is generalized by the GN model described above.

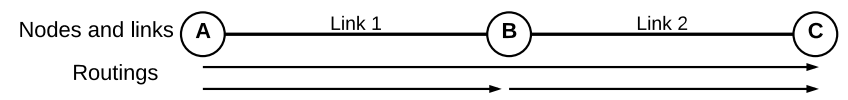

Fig. 1: The three-node network. The lengths of the two links are equal. Traffic flows from A to B, A to C, and B to C.

First, we compare the total bandwidth utilization for both schemes for a scenario with 6 spans per link, 5 AB channels, 5 B-C channels, and 6 A-C channels. By optimizing the channel powers and modulation formats, [4] achieved 2.45 Tbps throughput on each link using $525 \mathrm{GHz}$ of spectrum. The resulting allocation is shown in Fig. 2 (a), which closely resembles [4, Fig. 2 (c)]. Solving (3) for the obtained demands (as shown in Fig. 2 (a), 6 A-C channels use PM-16QAM with data rates of $200 \mathrm{Gbps}$, and $5 \mathrm{~A}-\mathrm{B}$ channels use PM-32QAM with $250 \mathrm{Gbps}$ ), with $\theta_{1}=1, \theta_{2}=$ $1 \times 10^{-3}, \theta_{3}=0$, our flexible grid optimization approach gives rise to Fig. 2 (b). We see that for the same throughput, the bandwidth usage is reduced to $325 \mathrm{GHz}$ (i.e., $38 \%$ bandwidth reduction). The gains come mainly from removing the guardband and, to some extent, from optimizing the modulation formats. Note that the channel spacing can be chosen according to the WSS, and not necessarily zero. The allocated PSDs and modulation formats are not as regular as the benchmark, because we have a larger solution space to search for the joint optimum.

Secondly, we investigate the impact of channel ordering on the bandwidth utilization. For the network in Fig. 1 with 6 channels for each source-destination pair, Fig. 3 (a) shows the optimized bandwidths of all orderings, normalized

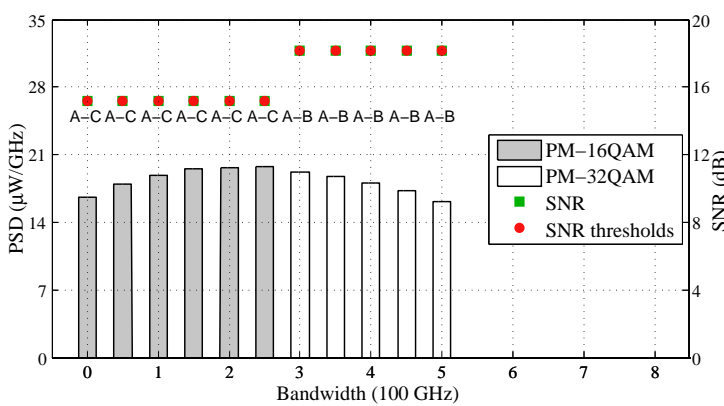

(a)

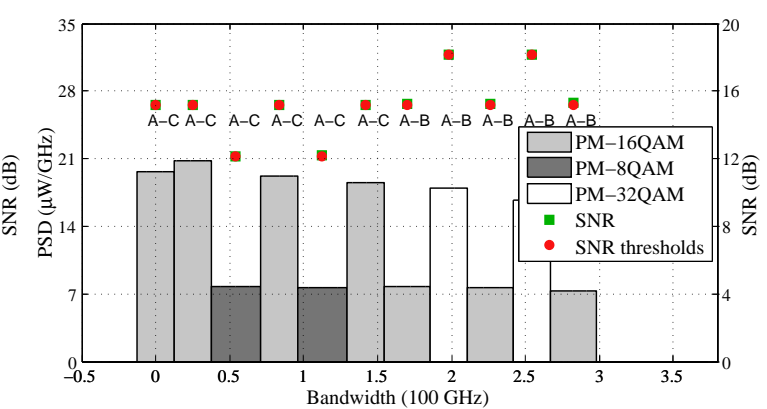

(b)

Fig. 2: Allocated spectra on link 1 where the traffic demands are $200 \mathrm{Gbps}$ for each of the 6 A-C channels, and $250 \mathrm{Gbps}$ for each of the 5 A-B channels. (a) The benchmark. (b) The proposed algorithm with the same channel orderings as in the benchmark. The SNRs are equal to the SNR thresholds for all the channels in both figures. 


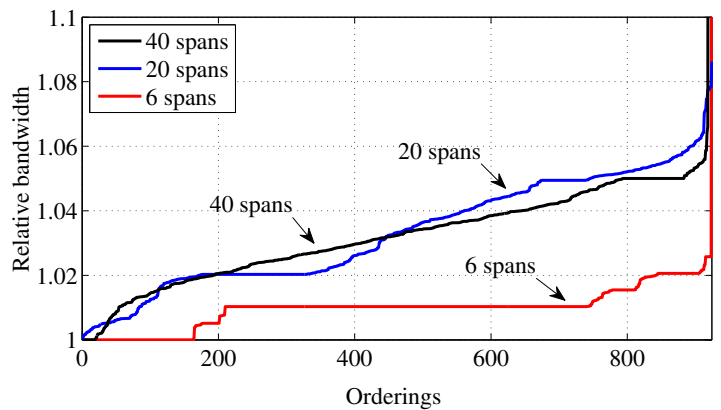

(a)

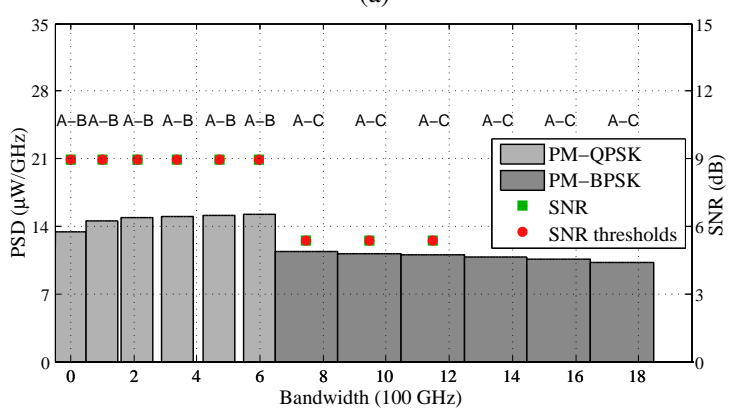

(c)

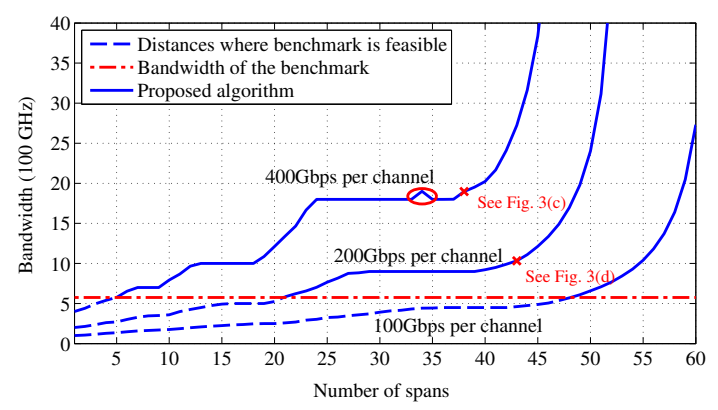

(b)

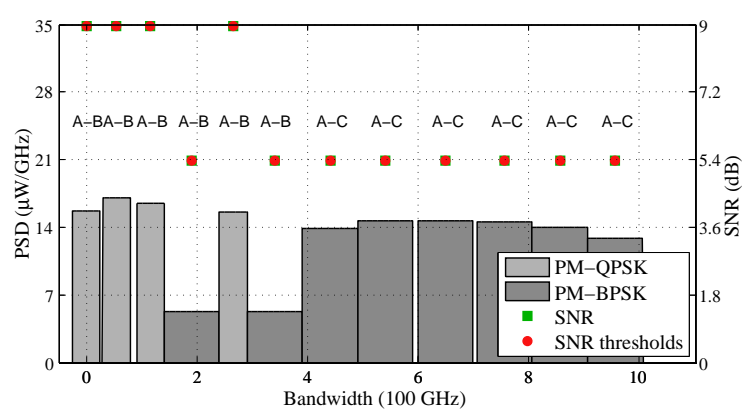

(d)

Fig. 3: (a) Normalized optimal bandwidths for all orderings. (b) Optimized bandwidth as a function of the length of each link for a fixed ordering. The blue dashed lines represent the distances where the benchmark is feasible. The benchmark always uses 575 GHz regardless of the data rate and distance. (c) Spectrum for link 1 with 38 spans per link and 400 Gbps per channel. (d) Spectrum for link 1 with 43 spans per link and 200 Gbps per channel. The SNRs are equal to the SNR thresholds for all the channels in Fig. 3 (c) and (d).

to the minimum bandwidth. We observe that in most cases, the variations are less than $6 \%$, indicating that, contrary to [4], our proposed algorithm is relatively insensitive to channel orderings. As the number of spans grows longer, the variation is slightly increased, and a small number of poor orderings (i.e., requiring relatively larger bandwidth) appear. However, such poor orderings can be avoided by grouping channels with the same source and destination together.

To further understand the impact of the link length, Fig. 3 (b) shows the optimized bandwidth as a function of the number of spans in each link for different data rates per channel. Each source-destination pair has 6 channels, whose spectral orderings are grouped together. The dashed part of the blue lines are the distances that the benchmark is feasible. As indicated by the red horizontal line, the benchmark uses $575 \mathrm{GHz}$ bandwidth regardless of the data rate and distance. For shorter distances, our algorithm requires less spectrum than the benchmark, while for longer distances, our algorithm still allows data transmission. The small peak at 34 spans is due to the unoptimized ordering. The allocated spectra of the crosses in Fig. 3 (b) are shown in Fig. 3 (c) and (d), respectively. In contrast to Fig. 2 (b), our optimization algorithm assigns different guardbands between channels to guarantee satisfactory SNRs.

\section{Conclusion}

We have presented a novel formulation to optimize any combination of bandwidth, power, and SNR margin, through optimal joint selection of modulation formats, carrier frequencies, and PSDs in flexible-grid optical networks. The optimization accounts for PLI through a GN model. While the formulation can be applied to any complex network with pre-determined routing, numerical results for a 3-node network indicate significant reductions in bandwidth and substantial increase in the overall throughput and transmission distance, compared to a fixed-grid WDM.

\section{References}

1. K. Christodoulopoulos, I. Tomkos, and E.A. Varvarigos, "Elastic Bandwidth Allocation in Flexible OFDM-based Optical Networks," J. Lightw. Technol. 29, 1354-1366 (2011).

2. P. Johannisson and E. Agrell, "Modeling of Nonlinear Signal Distortion in Fiber-Optical Networks," J. Lightw. Technol., to appear.

3. D. J. Ives, P. Bayvel, and S. J. Savory, "Adapting Transmitter Power and Modulation Format to Improve Optical Network Performance Utilizing the Gaussian Noise Model of Nonlinear Impairments," J. Lightw. Technol. 32, 3485-3494 (2014).

4. D. J. Ives and S. J. Savory. "Transmitter optimized optical networks," OFC, paper JW2A, (2013).

5. E. Palkopoulou et. al., "Nyquist-WDM-based Flexible Optical Networks: Exploring Physical Layer Design Parameters," J. Lightw. Technol. 31, 2332-2339 (2013).

6. O. Vassilieva et. al., "Systematic Analysis of Intra-Superchannel Nonlinear Crosstalk in Flexible Grid Networks," ECOC, paper Mo.4.3.6, (2014). 